\title{
Macronutrientes en el tejido foliar de albahaca Ocimum basilicum L. en respuesta a la aplicación de nitrógeno y potasio
}

\section{Macronutrients in the leaf tissue of basil Ocimum basilicum $\mathrm{L}$. in response to applications of nitrogen and potassium}

\author{
Enrique Combatt-Caballero ${ }^{1}$; Dairo Pérez-Polo ${ }^{2}$; José Villalba-Arteaga ${ }^{3}$; Jaime Mercado-Lázaro ${ }^{4}$; Alfredo Jarma-Orozco ${ }^{5}$
}

'Ing. Agrónomo, Ph.D. Universidad de Córdoba, Montería - Córdoba, Colombia; e-mail: ecombatt@correo.unicordoba.edu.co; (1) http://orcid.org/00000002-8760-0089

${ }^{2}$ Ing. Agrónomo, M.Sc. Universidad de Córdoba, Montería - Córdoba, Colombia; e-mail: dairoperezp@ @correo.unicordoba.edu.co; (1) http://orcid.org/00000001-9041-5856

3Ing. Agrónomo, M.Sc. Universidad de Córdoba, Montería - Córdoba, Colombia; e-mail: joseva984@gmail.com; (1) http://orcid.org/0000-0001-7679-8045

${ }^{4}$ Químico Puro, M.Sc. Universidad de Córdoba, Montería - Córdoba, Colombia; e-mail: jlmercadol@yahoo.es; (1) http://orcid.org/0000-0002-4177-1907

5Ing. Agrónomo, Ph.D. Universidad de Córdoba, Montería - Córdoba, Colombia; e-mail: jarma@fca.edu.co; (1) http://orcid.org/0000-0002-5821-2183

Cómo citar: Combatt-Caballero, E.; Pérez-Polo, D.; Villalba-Arteaga, J.; Mercado-Lázaro, J.; Jarma-Orozco, A. 2020. Macronutrientes en el tejido foliar de albahaca Ocimum basilicum L. en respuesta a la aplicación de nitrógeno y potasio. Rev. U.D.C.A Act. \& Div. Cient. 23(2):e1325. http://doi.org/10.31910/rudca.v23.n2.2020.1325

Artículo de acceso abierto publicado por Revista U.D.C.A Actualidad \& Divulgación Científica, bajo una licencia Creative Commons CC BY-NC 4.0

Publicación oficial de la Universidad de Ciencias Aplicadas y Ambientales U.D.C.A, Institución de Educación Superior Acreditada de Alta Calidad por el Ministerio de Educación Nacional.

Recibido: Agosto 31 de 2019 Aceptado: Octubre 29 de 2020 Editado por: Ingeborg Zenner de Polanía

\section{RESUMEN}

La albahaca (Ocimum basilicum L.) es un cultivo promisorio de reciente explotación y expansión en Colombia; sin embargo, esta tendencia se ve limitada por el desconocimiento de la nutrición con elementos, como $\mathrm{N}$ y K. Con la aplicación de dosis óptimas de $\mathrm{N}$ y K, los productores pueden incrementar la productividad y calidad de este sistema productivo, atrayendo nuevos productores. El objetivo de este estudio fue evaluar el efecto de la aplicación de nitrógeno y potasio sobre el contenido y relación de los macronutrientes en el tejido vegetal de albahaca. Las dosis de $\mathrm{N}$ y $\mathrm{K}$ fueron combinadas con base en la matriz Box-Berard aumentada (3): $2^{k}+2 k+2 k+1+1$, constituyendo un total de 14 tratamientos, dispuestos bajo un diseño completamente al azar, con tres repeticiones. Se estudió la masa seca (MS) y las relaciones N/K, N/P, N/S, N/Ca, K/Ca, K/ $\mathrm{Mg}, \mathrm{K} / \mathrm{P}$ y K/S, las cuales, fueron analizas con pruebas de medias y la metodología de superficie de respuesta. La albahaca desarrolló una mayor MS cuando la relación foliar de $\mathrm{N}$ y K con el resto de los nutrientes fue alta, a excepción de la relación de estos $(\mathrm{N} / \mathrm{K})$, la cual, fue estrecha. En este sentido, la MS fue $116,72 \%$ superior para las relaciones asociadas con nitrógeno y $120,84 \%$ para las de potasio, con respecto a las dosis mínimas de $\mathrm{N}$ y K. En albahaca, la concentración foliar de $\mathrm{N}$ y $\mathrm{K}$ debe predominar sobre el resto de macroelementos, condición obtenida con dosis de 100-190 $\mathrm{kg} \mathrm{ha}^{-1}$ de $\mathrm{N}$ y de $125-237,5 \mathrm{~kg} \mathrm{ha}^{-1}$ de $\mathrm{K}$.

Palabras clave: Tejido vegetal; Nutrición; Masa seca; Dosis. 


\section{ABSTRACT}

Basil (Ocimum basilicum L.) is a promising crop of recent exploitation and expansion in Colombia, however, this trend is limited by ignorance of nutrition requirements of elements such as $\mathrm{N}$ and $\mathrm{K}$. With the application of optimal doses of $\mathrm{N}$ and $\mathrm{K}$, producers can increase the productivity and quality of this production system, attracting new producers. The objective of this study was to evaluate the effect of the application of nitrogen and potassium on the content and ratio of macronutrients in basil plant tissue. The doses of $\mathrm{N}$ and $\mathrm{K}$ were combined based on the increased Box-Berard matrix (3): $2^{\mathrm{k}}+2 \mathrm{k}+2 \mathrm{k}+1+1$, constituting a total of 14 treatments, arranged under a completely randomized design with three repetitions. The dry mass (DM) and the relationships $\mathrm{N} / \mathrm{K}$, $\mathrm{N} / \mathrm{P}, \mathrm{N} / \mathrm{S}, \mathrm{N} / \mathrm{Ca}, \mathrm{K} / \mathrm{Ca}, \mathrm{K} / \mathrm{Mg}, \mathrm{K} / \mathrm{P}$ and $\mathrm{K} / \mathrm{S}$ were studied, and analyzed with tests of means and the response surface methodology. Basil developed a higher DM when the leaf ratio of $\mathrm{N}$ and $\mathrm{K}$ with the rest of the nutrients was high, except for the ratio of these $(\mathrm{N} / \mathrm{K})$, which was close. In this sense, the DM was $116.72 \%$ higher for the ratios associated with nitrogen and $120.84 \%$ for those of potassium, with respect to the minimum doses of $\mathrm{N}$ and $\mathrm{K}$. In basil, the foliar concentration of $\mathrm{N}$ and $\mathrm{K}$ should predominate over the rest of macroelements, a condition obtained with doses of 100 $190 \mathrm{~kg} \mathrm{ha}^{-1}$ of $\mathrm{N}$ and $125-237.5 \mathrm{~kg} \mathrm{ha}{ }^{-1}$ of $\mathrm{K}$.

Keywords: Plant tissue; Nutrition; Dry mass; Dosage.

\section{INTRODUCCIÓN}

El cultivo de albahaca Ocimum basilicum L. (Lamiaceae) es una alternativa agrícola para Colombia. Esta planta es una especie aromática que se caracteriza por su uso condimentario y farmacéutico, dadas su propiedades diuréticas y estimulantes, al igual que por su alto contenido de aceite esencial, a base de cineol, metilcahvicol, linalol, estragol, eugenol y timol, empleados en la industria de cosméticos, alimentos y fármacos (Benavides Mendoza et al. 2010; Moncayo et al. 2015).

Según Cortés \& Clavijo (2008), las especies aromáticas, tales como la albahaca, son cultivos promisorios en Colombia, con gran potencial económico, que cada vez atraen más productores por su reciente y creciente comercialización a mercados internacionales. En la actualidad, se producen 100t (Agronet, 2019), siendo los principales productores, los departamentos de Boyacá (72,97\%) y Tolima $(27,03 \%)$.

La reciente explotación y expansión de albahaca en Colombia enfrenta serias limitaciones tecnológicas (Bonilla \& Guerrero, 2010), entre las cuales, se encuentra la determinación de los requerimientos nutricionales óptimos del cultivo, necesarios para producir volúmenes constantes de biomasa, con altos estándares de calidad.

El manejo adecuado de nutrientes es esencial para el desarrollo de biomasa de los cultivos, ya que, si el suministro de nutrientes es deficiente, los rendimientos, se reducen (Pedraza \& Henao, 2008). En albahaca, Ferreira et al. (2016) y Yassue et al. (2018) reportan el incremento de la biomasa tras la aplicación de nitrógeno, con dosis cercanas a los $120 \mathrm{~kg} \mathrm{ha}^{-1}$. En este sentido, Malik et al. (2011) afirman que la fertilización es la mejor estrategia para mejorar los rendimientos y los compuestos activos de las plantas medicinales.

Entre los elementos nutricionales que requieren las plantas para su crecimiento y desarrollo, se encuentran el nitrógeno $(\mathrm{N})$ y potasio $(\mathrm{K})$, que forman parte del grupo de nutrientes esenciales de las plantas. El $\mathrm{N}$ tiene una función metabólica y estructural, hace parte de proteínas, moléculas de clorofila, ácidos nucleicos, que son fundamentales en procesos fisiológicos de absorción iónica, fotosíntesis, respiración y síntesis celular (Marschner, 2012). Según Robertson \& Vitousek (2009), para maximizar el crecimiento y el rendimiento de plantas medicinales es necesario conocer los niveles óptimos de fertilización, que permitan proveer al cultivo suficiente $\mathrm{N}$.

Por otra parte, el $\mathrm{K}+$ cumple las principales funciones fisiológicas, como controlar la translocación y la acumulación de azúcares, mantener el balance de cargas aniónicas a nivel celular y la regulación del estado hídrico de la planta; por eso es importante para la comprensión de la fisiología de los cultivos. Según Azcón \& Talón (2008), en la activación enzimática, el $\mathrm{K}+$ activa el metabolismo de muchas enzimas y proteínas, responsables del metabolismo de carbohidratos. La deficiencia de $\mathrm{K}$ en las plantas produce, a menudo, un crecimiento lento, disminución de tolerancia a la sequía, desarrollo de tallos débiles y una mayor susceptibilidad a enfermedades (Xu et al. 2011).

Pedraza \& Henao (2008) señalan que el análisis del tejido foliar es una herramienta esencial para su monitoreo y la posterior planificación de la fertilización, con el fin de relacionar esta información con la producción de cultivos. Nurzyńska et al. (2011) encontraron entre 2,82 y $4,34 \%$ de $\mathrm{N}$ y 2,26 y $4,18 \%$ de $\mathrm{K}$, como resultado del incremento de la dosis de nitrógeno y potasio en el cultivo de albahaca. La acumulación de potasio, principalmente en las hojas, suele ser mayor que la de otros macronutrientes; sin embargo, las concentraciones básicas de nutrientes y de elementos minerales son igualmente importantes para el contenido de sustancias bioactivas para la calidad de materiales, a base de hierbas (Dzida et al. 2015).

El objetivo de la presente investigación fue determinar el contenido y la relación de macronutrientes en el tejido foliar de plantas de albahaca en función de la aplicación de $\mathrm{N}$ y $\mathrm{K}$, bajo condiciones controladas.

\section{MATERIALES Y MÉTODOS}

Localización. Esta investigación, se realizó en las condiciones controladas de los umbráculos de la granja experimental de la Universidad de Córdoba. Esta zona, se caracteriza por una precipitación anual promedio de $1.346,1 \mathrm{~mm}$, humedad relativa del $84,0 \%$, temperatura promedio anual de $27,4^{\circ} \mathrm{C}$ y brillo solar anual de 2.108,2h. Según la clasificación de Holdridge, esta zona se clasifica como bosque seco tropical (bs-T) (Palencia et al. 2006). 
Diseño experimental. Las dosis de $\mathrm{N}$ y $\mathrm{K}$ fueron combinadas mediante la matriz Box-Berard aumentada (3): $2^{k}+2 k+2 k+1+1$ (Box, 1954), constituyendo un total de 14 tratamientos, conformados por siete dosis de nitrógeno y de potasio, siendo dispuestos bajo un diseño completamente al azar, con tres repeticiones.

Cada unidad experimental fue constituida por una planta de albahaca (O. basilicum) híbrido Nufar F1, establecida en una maceta con $8 \mathrm{~kg}$ de suelo típico del Valle del Sinú medio, con textura arcillo limosa, con las siguientes características químicas: $\mathrm{pH}(6,6)$; CICe $\left(21,9 \mathrm{cmol}(+) \mathrm{kg}^{-1}\right)$; materia orgánica $(2,96 \%)$; macronutrientes intercambiables: $\mathrm{Ca}, \mathrm{Mg}, \mathrm{K}$ y Na, con 13,2; 7,9; 0,55 y 0,2 cmol(+) $\mathrm{kg}^{-1}$, respectivamente; $\mathrm{S}$ y P disponible, con 10,4 y $12,6 \mathrm{mg} \mathrm{kg}^{-1} ; \mathrm{y}$ micronutrientes intercambiables: $\mathrm{Cu}, \mathrm{Fe}, \mathrm{Zn}, \mathrm{Mn}$ y $\mathrm{B}$ con 2,8; 4,0; 60,8 y $0,28 \mathrm{mg} \mathrm{kg}^{-1}$, de acuerdo con los resultados analíticos del Laboratorio de Suelos y Aguas de la Universidad de Córdoba.

La fertilización fue realizada, a través de la aplicación de soluciones nutritivas. El N, se suministró en forma de $\mathrm{NH}_{4}{ }^{+}$y fraccionado en tres partes iguales, a los 10, 44 y 77 días después del trasplante. El $\mathrm{K}$ fue aplicado en forma de $\mathrm{KCl}$ y fraccionado en dos aplicaciones iguales, que coincidieron con la primera y la segunda fertilización de $\mathrm{N}$; con el fin de evitar deficiencias nutricionales que pudieran influir sobre la respuesta de $\mathrm{N}$ y K, se realizó una aplicación uniforme de fósforo $(\mathrm{P})$ fraccionada en dos partes iguales: la primera, un día antes del trasplante y la segunda, con la primera aplicación de $\mathrm{N}$ y $\mathrm{K}$. Adicionalmente, se utilizó la solución de micronutrientes de Ruiz (1997), aplicada en la primera y segunda aplicación de N y K.

Cuando las plantas alcanzaron el punto de cosecha fueron extraídas por completo y deshidratadas, para la determinación de masa seca (MS). El análisis de tejido vegetal, se realizó sobre una muestra secada en estufa de circulación forzada, a temperatura de $70^{\circ} \mathrm{C}$, por 72 horas y molida en un micro-molino IKA MSF-1, con malla de $1 \mathrm{~mm}$.

Para la determinación del contenido de elementos nutricionales Ca, Mg y K, las muestras vegetales fueron sometidas en una digestión vía húmeda, con $\mathrm{HNO}_{3}: \mathrm{HClO}_{4}$ en relación 3:1 v/v, a una temperatura de $350^{\circ} \mathrm{C}$ y cuantificados por espectroscopia atómica, en un equipo Perkin Elmer 3110. El nitrógeno, se cuantificó por el método de Kjeldahl; el fósforo, por el método colorimétrico, con molibdato de amonio y el azufre, por turbidimetría con cloruro de bario $\left(\mathrm{BaCl}_{2}\right)$, según el Instituto Geográfico Agustín Codazzi (IGAC, 2006). Con los nutrientes cuantificados fueron analizadas las siguientes relaciones entre nutrientes en el tejido vegetal: $\mathrm{N} / \mathrm{K}, \mathrm{N} / \mathrm{P}, \mathrm{N} / \mathrm{S}$, $\mathrm{N} / \mathrm{Ca}, \mathrm{K} / \mathrm{Ca}, \mathrm{K} / \mathrm{Mg}, \mathrm{K} / \mathrm{P}$ y K/S.

Análisis de datos. Con los datos, se realizó una prueba de comparación de medias de Tukey $(p=0,05)$. Además, el efecto del $\mathrm{N}$ y K sobre las relaciones macronutrientes fue estimado con las ecuaciones de regresión lineal múltiple de mejor ajuste, en el programa R (Development Core Team, versión 3.5.3, 2019). Estos modelos fueron graficados con la metodología de superficie de respuesta en el programa SigmaPlot versión 10.10. Por otra parte, se determinó el coeficiente de correlación de Pearson entre la masa seca foliar y las relaciones de nutrientes en el tejido vegetal.

\section{RESULTADOS Y DISCUSIÓN}

En la tabla 1, se muestran los contenidos de macronutrientes en el tejido vegetal de albahaca, donde los elementos de mayor concentración fueron $\mathrm{N}, \mathrm{K}$ y Ca, con valores de hasta 3,32, 3,84 y $1,47 \%$, respectivamente. Los contenidos foliares de $\mathrm{N}$ y $\mathrm{K}$ fueron superiores a los reportados por Rao et al. (2007) en albahaca, que oscilaron entre 1,36 y $1,81 \%$, para $\mathrm{N}$ y de 1,05 a $2,06 \%$, para $\mathrm{K}$ y, a diferencia de estos autores, respondieron a la aplicación edáfica de estos nutrientes; sin embargo, estos contenidos fueron inferiores a los registrados por Nurzyńska et al. (2011), cuya concentración varió entre 2,82 y 4,34\%, de $\mathrm{N}$ y de 2,26 a $4,18 \%$, de $\mathrm{K}$, como resultado del incremento de la dosis de nitrógeno y potasio, en el cultivo de albahaca.

En cuanto a la MS, la máxima ganancia por planta $(50,38 g)$, se registró con el T11, que correspondió a la mayor dosis de $\mathrm{N}$ y una de las dosis más altas de K (Tabla 1). La concentración de elementos químicos en el tejido foliar depende de la ganancia de MS de cada tratamiento, ya que, a medida que hay mayor formación de estructuras vegetativas, se puede presentar un efecto de dilución de los mismos, por lo tanto, menor contenido de algunos elementos químicos. De acuerdo con Mapeli et al. (2005), la nutrición mineral es importante, dado que la deficiencia o exceso de nutrientes interfiere en la producción de biomasa y en la cantidad de principios activos, contenidos en los extractos vegetales.

Relaciones entre el nitrógeno con potasio, fósforo, azufre y calcio. En N/K, la mayor producción de biomasa estuvo asociada con una relación estrecha de estos elementos, de tal forma que, el T2, T4, T8, T11 y T12, produjeron una MS promedio de 44,02g, casi el doble de la obtenida con las dosis mínimas de N y K (T14), donde hubo una alta relación (Tablas 1 y 2). Esta respuesta sugiere que la eficiencia en la producción de MS de albahaca se encuentra asociada con una relación sinérgica entre los contenidos foliares de $\mathrm{N}$ y $\mathrm{K}$, asimismo, que esta condición se alcanza con la aplicación de una dosis mínima de $100 \mathrm{~kg} \mathrm{ha}^{-1}$, de N y entre 187,5-237,5kg ha ${ }^{-1}$, de K. Estos resultados son similares a los reportes de Sharafzadeh \& Alizadeh (2011) en albahaca, quienes señalan que el rendimiento responde a la fertilización con dosis de 104 a 200kg/ha, de $\mathrm{N}$ y de 73 a $120 \mathrm{~kg} / \mathrm{ha}$, de K; también, con Ferreira et al. (2016), donde se elevaron las características productivas del cultivo, con dosis de 90 a $135 \mathrm{~kg} \mathrm{ha}^{-1}$ de $\mathrm{N}$.

Según Frabboni et al. (2011), la biomasa de albahaca es favorecida por dosis de 160 a $240 \mathrm{~kg} \mathrm{ha}^{-1}$, de N. La alta demanda de nitrógeno en este cultivo (fotosíntesis tipo C3), se explica, según Azcón \& Talón (2008), por la importancia de este nutriente en la síntesis de la enzima RuBisCo (ribulosa 1,5 bifosfato carboxilasa-oxigenasa) en la hoja, responsable de la fijación de $\mathrm{CO}_{2}$ en la fotosíntesis; es así que, hojas con una concentración optima de nitrógeno, serán más eficientes en la producción de biomasa, dado que experimentarán una mayor tasa de fotosíntesis. Por su parte, la demanda de potasio, se explica por su función en la activación y síntesis de ATP, así como de proteínas asociadas con la fijación de $\mathrm{CO}_{2}$ que, en cantidades considerables en las estomas, pueden mantener altas tasas de fotosíntesis (Marschner, 2012). 
Tabla 1. Contenido de macronutrientes en tejidos vegetales en hoja de albahaca, bajo el efecto de 14 dosis de N y K, según la matriz BoxBerard aumentada (3): $2^{\mathrm{k}}+2 \mathrm{k}+2 \mathrm{k}+1+1$ (Box, 1954).

\begin{tabular}{|c|c|c|c|c|c|c|c|c|c|}
\hline \multirow{2}{*}{ Trat. } & $\mathbf{N}$ & $\mathbf{K}$ & $\mathbf{N}$ & $\mathbf{P}$ & $\mathbf{K}$ & $\mathrm{Ca}$ & $\mathbf{M g}$ & S & \multirow{2}{*}{$\begin{array}{c}\text { MS } \\
\mathrm{g}\end{array}$} \\
\hline & \multicolumn{2}{|c|}{$\mathrm{kg} \mathrm{ha}^{-1}$} & \multicolumn{6}{|c|}{---------------------------------- \% } & \\
\hline 1 & 50,0 & 62,5 & 1,90 & 0,29 & 2,20 & 1,47 & 0,36 & 0,09 & 23,62 def \\
\hline 2 & 50,0 & 187,5 & 2,42 & 0,27 & 2,48 & 1,14 & 0,35 & 0,10 & $39,52 \mathrm{c}$ \\
\hline 3 & 150,0 & 62,5 & 2,43 & 0,25 & 1,99 & 1,21 & 0,35 & 0,04 & $39,29 \mathrm{c}$ \\
\hline 4 & 150,0 & 187,5 & 2,50 & 0,28 & 2,54 & 0,82 & 0,29 & 0,10 & $43,64 \mathrm{abc}$ \\
\hline 5 & 25,0 & 125,0 & 2,30 & 0,31 & 2,16 & 1,13 & 0,36 & 0,11 & $26,24 \mathrm{de}$ \\
\hline 6 & 175,0 & 125,0 & 2,06 & 0,25 & 2,24 & 1,01 & 0,33 & 0,06 & $48,46 \mathrm{ab}$ \\
\hline 7 & 100,0 & 31,2 & 3,12 & 0,30 & 2,20 & 1,16 & 0,40 & 0,12 & $31,22 \mathrm{~d}$ \\
\hline 8 & 100,0 & 218,7 & 2,34 & 0,28 & 3,79 & 1,19 & 0,34 & 0,14 & $39,08 \mathrm{c}$ \\
\hline 9 & 10,0 & 62,5 & 2,51 & 0,34 & 2,59 & 1,34 & 0,37 & 0,20 & $17,75 \mathrm{fg}$ \\
\hline 10 & 50,0 & 12,5 & 1,66 & 0,32 & 1,95 & 1,13 & 0,36 & 0,13 & $22,02 \mathrm{efg}$ \\
\hline 11 & 190,0 & 187,5 & 2,42 & 0,32 & 3,84 & 1,02 & 0,32 & 0,18 & $50,38 \mathrm{a}$ \\
\hline 12 & 150,0 & 237,5 & 2,48 & 0,18 & 2,56 & 1,13 & 0,32 & 0,06 & $47,48 \mathrm{ab}$ \\
\hline 13 & 100,0 & 125,0 & 2,48 & 0,25 & 2,54 & 0,76 & 0,25 & 0,11 & $41,67 \mathrm{bc}$ \\
\hline 14 & 10,0 & 12,5 & 1,85 & 0,26 & 1,60 & 1,00 & 0,32 & 0,13 & $16,16 \mathrm{~g}$ \\
\hline
\end{tabular}

Trat: Tratamiento; *: Letras similares en sentido vertical no difieren estadísticamente, según la prueba de Tukey $(\mathrm{p}=0,05)$.

Tabla 2. Valores medios de la relación entre contenidos de nitrógeno con potasio, fósforo, azufre y calcio en tejido de albahaca, en función de la aplicación de combinaciones de dosis de $\mathrm{N}$ y K.

\begin{tabular}{|c|c|c|l|l|l|l|}
\hline \multirow{2}{*}{ Trat. } & \multicolumn{2}{|c|}{ Dosis $\left(\mathbf{k g ~ h a ~}^{-1}\right)$} & \multirow{2}{*}{$\mathbf{N} / \mathbf{K}$} & $\mathbf{N} / \mathbf{P}$ & $\mathbf{N}$ & \multirow{2}{*}{$\mathbf{N} / \mathbf{C} \mathbf{}$} \\
\cline { 2 - 5 } & $\mathbf{N}$ & $\mathbf{K}$ & & & & \\
\hline 1 & 50,0 & 62,5 & $0,03 \mathrm{de}^{*}$ & $6,46 \mathrm{de}$ & $20,33 \mathrm{def}$ & $1,29 \mathrm{f}$ \\
\hline 2 & 50,0 & 187,5 & $0,01 \mathrm{f}$ & $9,09 \mathrm{bcd}$ & $25,08 \mathrm{cde}$ & $2,13 \mathrm{cde}$ \\
\hline 3 & 150,0 & 62,5 & $0,04 \mathrm{~d}$ & $9,62 \mathrm{bc}$ & $60,93 \mathrm{a}$ & $2,02 \mathrm{cdef}$ \\
\hline 4 & 150,0 & 187,5 & $0,01 \mathrm{f}$ & $8,86 \mathrm{bcd}$ & $25,95 \mathrm{~cd}$ & $3,05 \mathrm{ab}$ \\
\hline 5 & 25,0 & 125,0 & $0,02 \mathrm{ef}$ & $7,53 \mathrm{cde}$ & $21,35 \mathrm{def}$ & $2,09 \mathrm{cdef}$ \\
\hline 6 & 175,0 & 125,0 & $0,02 \mathrm{ef}$ & $8,29 \mathrm{bcd}$ & $32,40 \mathrm{bc}$ & $2,04 \mathrm{cdef}$ \\
\hline 7 & 100,0 & 31,2 & $0,10 \mathrm{c}$ & $10,51 \mathrm{~b}$ & $26,37 \mathrm{~cd}$ & $2,69 \mathrm{abc}$ \\
\hline 8 & 100,0 & 218,8 & $0,01 \mathrm{f}$ & $8,50 \mathrm{bcd}$ & $16,39 \mathrm{def}$ & $2,05 \mathrm{cdef}$ \\
\hline 9 & 10,0 & 62,5 & $0,04 \mathrm{~d}$ & $7,41 \mathrm{cde}$ & $12,70 \mathrm{f}$ & $1,87 \mathrm{cdef}$ \\
\hline 10 & 50,0 & 12,5 & $0,13 \mathrm{~b}$ & $5,25 \mathrm{e}$ & $12,85 \mathrm{f}$ & $1,48 \mathrm{ef}$ \\
\hline 11 & 190,0 & 187,5 & $0,01 \mathrm{f}$ & $7,61 \mathrm{cde}$ & $13,51 \mathrm{f}$ & $2,39 \mathrm{bcd}$ \\
\hline 12 & 150,0 & 237,5 & $0,01 \mathrm{f}$ & $13,53 \mathrm{a}$ & $41,03 \mathrm{~b}$ & $2,20 \mathrm{cde}$ \\
\hline 13 & 100,0 & 125,0 & $0,02 \mathrm{ef}$ & $9,72 \mathrm{bc}$ & $22,72 \mathrm{cdef}$ & $3,31 \mathrm{a}$ \\
\hline 14 & 10,0 & 12,5 & $0,15 \mathrm{a}$ & $7,12 \mathrm{cde}$ & $14,74 \mathrm{ef}$ & $1,86 \mathrm{def}$ \\
\hline
\end{tabular}

Trat.: Tratamiento; *: Letras similares en sentido vertical no difieren estadísticamente, según la prueba de Tukey $(p=0,05)$. 
En la figura $1 \mathrm{~A}$, se observa que la relación $\mathrm{N} / \mathrm{K}$ depende principalmente de $\mathrm{K}$, ya que, al aumentar las dosis de este elemento, disminuye la relación hasta volverse estrecha, donde se produce la máxima biomasa, respuesta que coincide con lo registrado en los tratamientos T2, T4, T8, T11 y T12.

La relación N/P registró la mayor MS con el T12, cuyo valor fue uno de los más altos, superando en $115,62 \%$ la producida por el T10, donde hubo la menor relación en el tejido vegetal (Tablas 1 y 2). Estos resultados indican que la producción de biomasa de este cultivo demanda de una alta concentración foliar de nitrógeno y que estos contenidos deben ser hasta 13 veces mayores que los de fósforo, condición que se alcanzó con la aplicación de $150 \mathrm{~kg} \mathrm{ha}^{-1}$, de $\mathrm{N}$ y la mayor dosis, de K. Si bien los contenidos foliares de fósforo son mínimos, la función de este nutriente no puede ser sustituida por otro, ya que, según Reichardt \& Timm (2004), participa en procesos vitales, como la formación estructural de las plantas, el suministro de energía para la producción de foto asimilados y la calidad del producto final.

En la figura 1B, se observa que al incrementar las dosis $\mathrm{K}$ ocurre una menor absorción de P foliar, pero a dosis mayores de $100,0 \mathrm{~kg}$ $\mathrm{ha}^{-1}$ de $\mathrm{N}$, la relación $\mathrm{N} / \mathrm{P}$ disminuye, lo que indica que disminuye la concentración de N, a nivel de tejido foliar. Rao et al. (2007) reportaron que los contenidos foliares de $\mathrm{P}$ tendieron a disminuir con las dosis de nitrógeno, siendo esta respuesta explicada por el efecto dilución, que representa el incremento de los rendimientos de la hierba. Similares efectos de dilución han sido observados en citronela (Puttanna et al. 2001); sin embargo, Biesiada \& Kús (2010) reportan altas cantidades de fósforo en plantas de albahaca, con dosis de $150 \mathrm{~kg} \mathrm{ha}^{-1}$, de $\mathrm{N}$ y Shuman (1994) reportaran que el fósforo y el nitrógeno interactúan de forma sinérgica y en dosis adecuadas, su efecto conjunto es mejor para la producción vegetal, que cuando son aplicados separadamente.

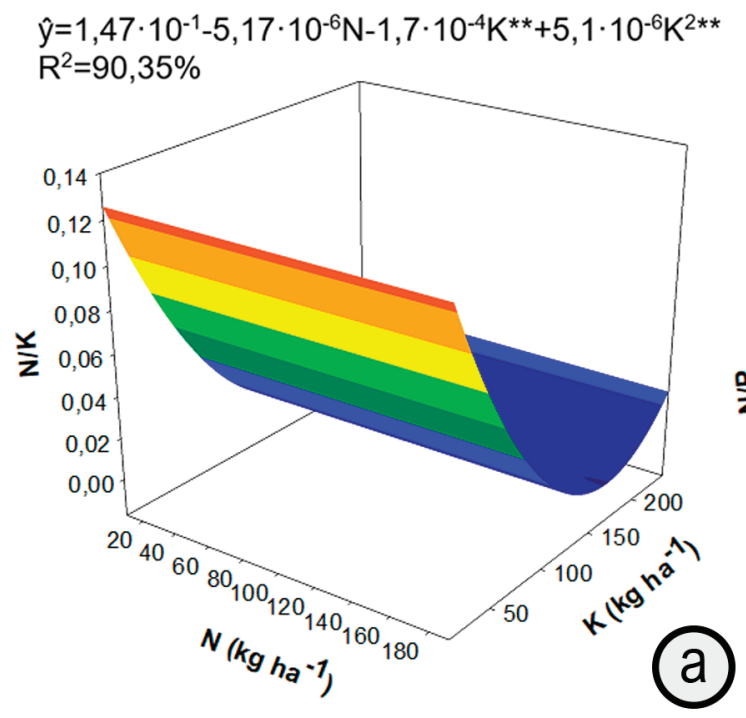

\section{$\hat{y}=5,46+4,9 \cdot 10^{-2} N^{*}+8,6 \cdot 10^{-3} K^{*}-2,04 \cdot 10^{-4} \mathrm{~N}^{2 *}$ \\ $R^{2}=35,61 \%$}
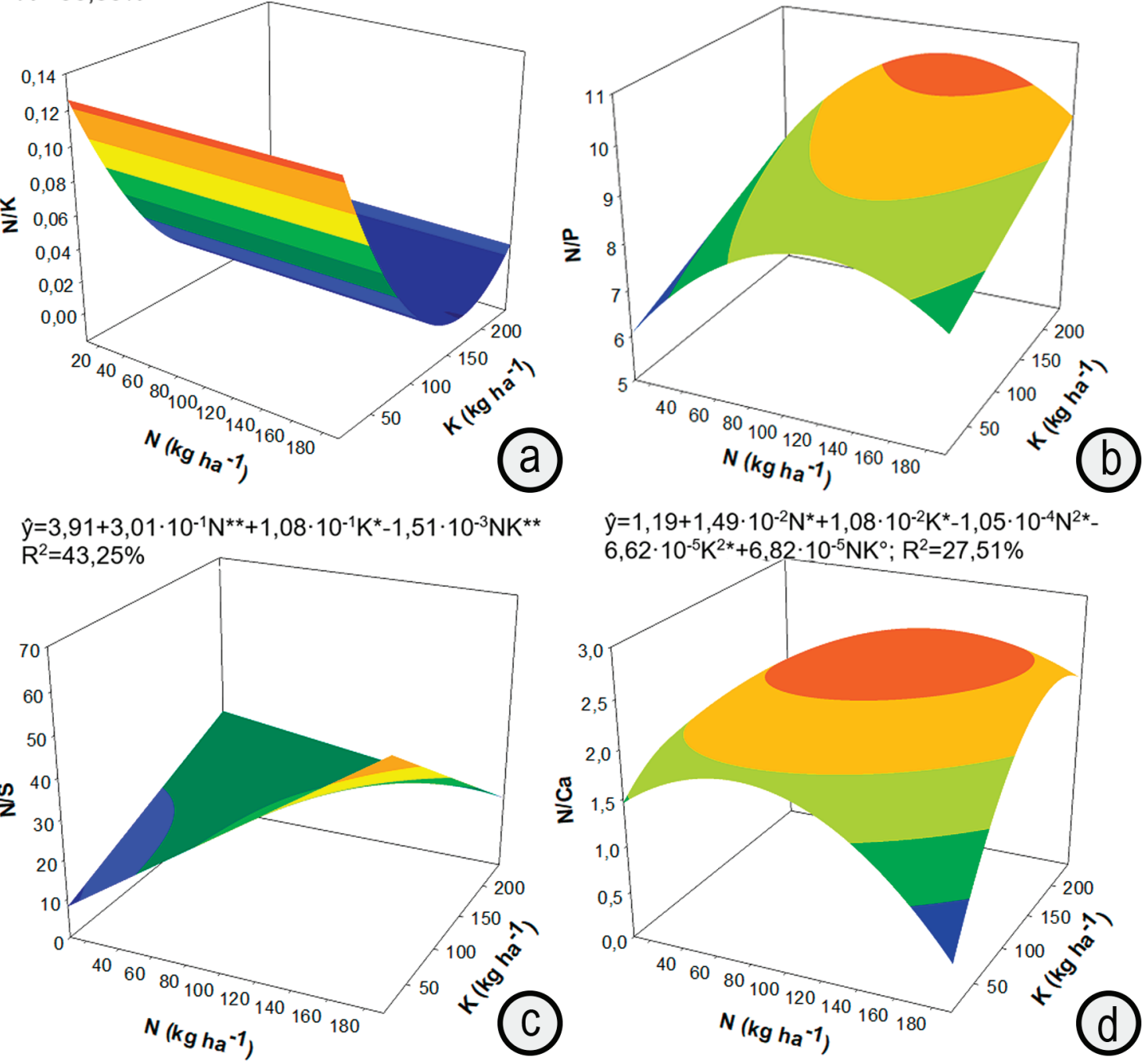

$\hat{\mathrm{y}}=1,19+1,49 \cdot 10^{-2} \mathrm{~N}^{*}+1,08 \cdot 10^{-2} \mathrm{~K}^{\star}-1,05 \cdot 10^{-4} \mathrm{~N}^{2 *}-$

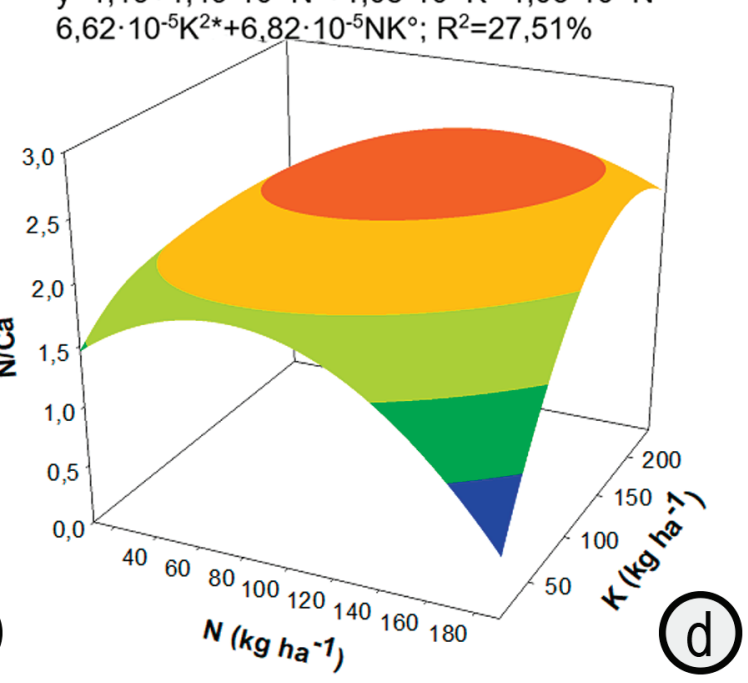

Figura 1. Efecto de dosis de $\mathrm{N}$ y K en la relación entre $\mathrm{N}$ con: a. potasio; b. fósforo; c. azufre; d. calcio, en el tejido vegetal de albahaca. 
En N/S, las mayores relaciones en el tejido foliar $(44,79$, en promedio), se encontraron en el T3, T6 y T12, donde la MS promedio fue de $45,08 \mathrm{~g}$, la cual, superó en $153,97 \%$ los valores registrados por el T9, cuya relación fue la menor (Tablas 1 y 2). Esta respuesta es similar a la observada en N/P, pero, en este caso, se requiere de una concentración foliar de $\mathrm{N}$ casi 45 veces la de $\mathrm{S}$. Esta relación fue superior a la encontrada por Orman (2012), en el cultivo de tomate, que osciló entre 3,18 y 4,17. Por otra parte, De Souza et al. (2014) obtuvieron mayor productividad de tomate, con la relación $\mathrm{N} / \mathrm{S}$, de 2,36.

En la figura $1 \mathrm{C}$, se aprecia que la relación $\mathrm{N} / \mathrm{S}$ se incrementa linealmente con la aplicación de $\mathrm{N}$ a bajas dosis de $\mathrm{K}$, pero esta tiende a disminuir, cuando se aumenta la dosis de este último, respuesta que sugiere un antagonismo de $\mathrm{K}$ sobre la relación $\mathrm{N} / \mathrm{S}$, que favorece la acumulación foliar de S. De acuerdo con Malavolta \& Moraes (2007), el N y el S pueden sufrir sinergismos e influenciar en la calidad de los productos y según Conley et al. (2002), cuando el contenido de $\mathrm{N}$ en las hojas es bajo, las plantas no metabolizan nutrientes eficientemente, ni usan eficientemente el S.

Para la relación N/Ca, los mayores valores se encontraron con el T4 y T13, que tuvieron una biomasa promedio de $42,65 \mathrm{~g}$ y superaron en $80,57 \%$, la producida por el T1, cuya relación fue la más inferior (Tablas 1 y 2). La respuesta de N/Ca fue similar a la observada en $\mathrm{N} / \mathrm{P}$ y N/S, pero, en este caso, a nivel foliar, se requiere una concentración de $\mathrm{N}$ superior hasta tres veces la de Ca; asimismo, esta condición se alcanzó con la aplicación de dosis entre medias $\mathrm{y}$ altas de $\mathrm{N}$ y K.
La relación $\mathrm{N} /$ Ca reportó efectos significativos para los componentes lineales y cuadráticos de $\mathrm{N}$ y $\mathrm{K}$, donde se puede apreciar que la relación N/Ca aumenta conforme se incrementa la dosis de $\mathrm{N}$ y $\mathrm{K}$ hasta alcanzar su máximo valor, a partir de donde comienza a decrecer (Figura 1D). Según Bar-Tal et al. (2001), la disminución de Ca foliar se explica por la sobreoferta de $\mathrm{N}$, especialmente, con mayores relaciones $\mathrm{NH}_{4}: \mathrm{NO}_{3}$, que reduce la absorción de calcio; resultados que difieren con lo reportado por Biesiada \& Kús (2010), en albahaca, donde los contenidos foliares de calcio aumentaron con la adición de $150 \mathrm{~kg} \mathrm{ha}^{-1}$ de $\mathrm{N}$.

Relaciones entre potasio con calcio, magnesio, fósforo y azufre. En $\mathrm{K} / \mathrm{Ca}$, la mayor relación se obtuvo con el T11 y T13, cuya MS media fue de 46,02g, que superó en $82,11 \%$ la alcanzada por el T1, T3, T10 y T14, donde la relación de nutrientes fue la menor, con un valor medio de 1,63 (Tablas 1 y 3). Los resultados indican que, para alcanzar una alta producción de biomasa, los contenidos foliares de potasio deben predominar hasta 3,6 veces sobre los de calcio; asimismo, para obtener este estado en la hoja, se deben adicionar dosis medias o altas de N y K. Esta respuesta difiere con Nurzyńska et al. (2012), quienes encontraron que los contenidos de Ca se incrementan en plantas de albahaca, con la aplicación de dosis adecuadas de $\mathrm{N}$ y K.

La relación $\mathrm{K} / \mathrm{Ca}$ disminuye a bajas dosis de $\mathrm{K}$ e incrementos de $\mathrm{N}$; no obstante, aumenta conforme se adiciona $\mathrm{K}$, de tal manera, que el máximo contenido de $\mathrm{K}$ foliar sobre Ca se obtiene con las mayores dosis de N y K (Figura 2A). Según Nukaya et al. (1995), esta respuesta obedece a que los altos niveles de potasio en el entorno de las raíces interfieren con la absorción de calcio.

Tabla 3. Valores medios de la relación entre contenidos de potasio con calcio, magnesio, fósforo y azufre en tejido de albahaca, en función de la aplicación de combinaciones de dosis de $\mathrm{N}$ y K.

\begin{tabular}{|c|c|c|l|l|l|l|}
\hline \multirow{2}{*}{ Trat. } & \multicolumn{2}{|c|}{ Dosis $\left(\mathbf{k g ~ h a}^{-1}\right)$} & \multirow{2}{*}{$\mathbf{K} / \mathbf{C a}$} & $\mathbf{K} / \mathbf{M g}$ & \multirow{2}{*}{$\mathbf{K} / \mathbf{P}$} & \multirow{2}{*}{$\mathbf{K} / \mathbf{S}$} \\
\cline { 2 - 3 } & $\mathbf{N}$ & $\mathbf{K}$ & & & & \\
\hline 1 & 50,0 & 62,5 & $1,49 \mathrm{~d} *$ & $6,21 \mathrm{de}$ & $7,48 \mathrm{c}$ & $23,41 \mathrm{cdef}$ \\
\hline 2 & 50,0 & 187,5 & $2,18 \mathrm{bcd}$ & $7,02 \mathrm{de}$ & $9,29 \mathrm{bc}$ & $25,84 \mathrm{cde}$ \\
\hline 3 & 150,0 & 62,5 & $1,67 \mathrm{~d}$ & $5,73 \mathrm{de}$ & $7,89 \mathrm{c}$ & $49,93 \mathrm{a}$ \\
\hline 4 & 150,0 & 187,5 & $3,10 \mathrm{abc}$ & $8,71 \mathrm{abcd}$ & $9,02 \mathrm{bc}$ & $26,49 \mathrm{~cd}$ \\
\hline 5 & 25,0 & 125,0 & $1,96 \mathrm{~cd}$ & $5,93 \mathrm{de}$ & $6,97 \mathrm{c}$ & $19,30 \mathrm{def}$ \\
\hline 6 & 175,0 & 125,0 & $2,23 \mathrm{bcd}$ & $6,82 \mathrm{de}$ & $9,02 \mathrm{bc}$ & $35,25 \mathrm{bc}$ \\
\hline 7 & 100,0 & 31,2 & $1,91 \mathrm{~cd}$ & $5,50 \mathrm{de}$ & $7,46 \mathrm{c}$ & $18,73 \mathrm{def}$ \\
\hline 8 & 100,0 & 218,8 & $3,32 \mathrm{ab}$ & $11,12 \mathrm{ab}$ & $13,77 \mathrm{a}$ & $26,56 \mathrm{~cd}$ \\
\hline 9 & 10,0 & 62,5 & $1,94 \mathrm{~cd}$ & $7,13 \mathrm{cde}$ & $7,65 \mathrm{c}$ & $13,15 \mathrm{ef}$ \\
\hline 10 & 50,0 & 12,5 & $1,74 \mathrm{~d}$ & $5,46 \mathrm{e}$ & $6,15 \mathrm{c}$ & $15,06 \mathrm{def}$ \\
\hline 11 & 190,0 & 187,5 & $3,79 \mathrm{a}$ & $11,93 \mathrm{a}$ & $12,06 \mathrm{ab}$ & $21,39 \mathrm{def}$ \\
\hline 12 & 150,0 & 237,5 & $2,27 \mathrm{bcd}$ & $7,92 \mathrm{bcde}$ & $14,00 \mathrm{a}$ & $42,94 \mathrm{ab}$ \\
\hline 13 & 100,0 & 125,0 & $3,41 \mathrm{ab}$ & $10,30 \mathrm{abc}$ & $9,97 \mathrm{abc}$ & $23,10 \mathrm{cdef}$ \\
\hline 14 & 10,0 & 12,5 & $1,62 \mathrm{~d}$ & $5,16 \mathrm{e}$ & $6,25 \mathrm{c}$ & $12,49 \mathrm{f}$ \\
\hline
\end{tabular}

Trat.: Tratamiento; *: Letras similares en sentido vertical no difieren estadísticamente, según la prueba de Tukey ( $\mathrm{p}=0,05)$. 
En $\mathrm{K} / \mathrm{Mg}$, la mayor relación se obtuvo con el T11, que superó en 163,91\% la MS registrada por el T10 y T14, cuya relación fue la más inferior, siendo de 5,31, en promedio (Tablas 1 y 3). Los resultados indican que en esta relación al igual que para $\mathrm{K} / \mathrm{Ca}$, los contenidos de potasio foliar deben ser superiores, en este caso, casi cuatro veces sobre magnesio. Este estado, se alcanza con la aplicación de altas dosis de N y K; sin embargo, esta respuesta contrasta la de Nurzyńska et al. (2012), donde el contenido de Mg foliar de albahaca se incrementó con la adición de dosis adecuadas de $\mathrm{N}$ y K. Por su parte, Marschner (2012), afirma que las deficiencias de $\mathrm{Mg}^{+2}$ en la planta ocurren por el incremento en las concentraciones de $\mathrm{K}^{+} \mathrm{y} \mathrm{Ca}^{2+}$, en la solución del suelo.

Los efectos significativos de $\mathrm{K} / \mathrm{Mg}$, para el componente lineal de $\mathrm{N}$, la interacción de $\mathrm{K}$ cuadrático y $\mathrm{N}$ lineal, así como la interacción $\mathrm{N} * \mathrm{~K}$ fueron similares a los observados en la relación $\mathrm{K} / \mathrm{Ca}$ (Figura 2B); sin embargo, una advertencia observada por Rosolem (2005), sostiene que la disminución de Ca y Mg no es una limitación para el crecimiento o la producción.

En K/P, los máximos valores se encontraron con el T8, T11 y T12, donde la MS promedio fue de 45,65g, superando en 81,41\%, la registrada por el T1, T3, T5, T7, T9, T10 y T14, cuya relación fue la más inferior, con 7,12, en promedio (Tabla 1 y 3). Al igual que para $\mathrm{K} / \mathrm{Ca}$ y $\mathrm{K} / \mathrm{Mg}$, en esta relación para una mayor eficiencia en la producción de biomasa, se requieren de altas cantidades de $\mathrm{K}$ en el tejido, donde esta concentración debe ser, aproximadamente, 13 veces la de fósforo. Esta condición tendió a alcanzarse con dosis altas de $\mathrm{K}$ y dosis medias o altas de $\mathrm{N}$, resultados que difieren con Rao et al. (2007), quienes reportaron que los contenidos foliares de P tendieron a no responder a la aplicación de K y de Nurzyńska et al. (2012), quienes encontraron que los contenidos de P en albahaca se incrementan con la aplicación de dosis adecuadas de $\mathrm{N}$ y K.
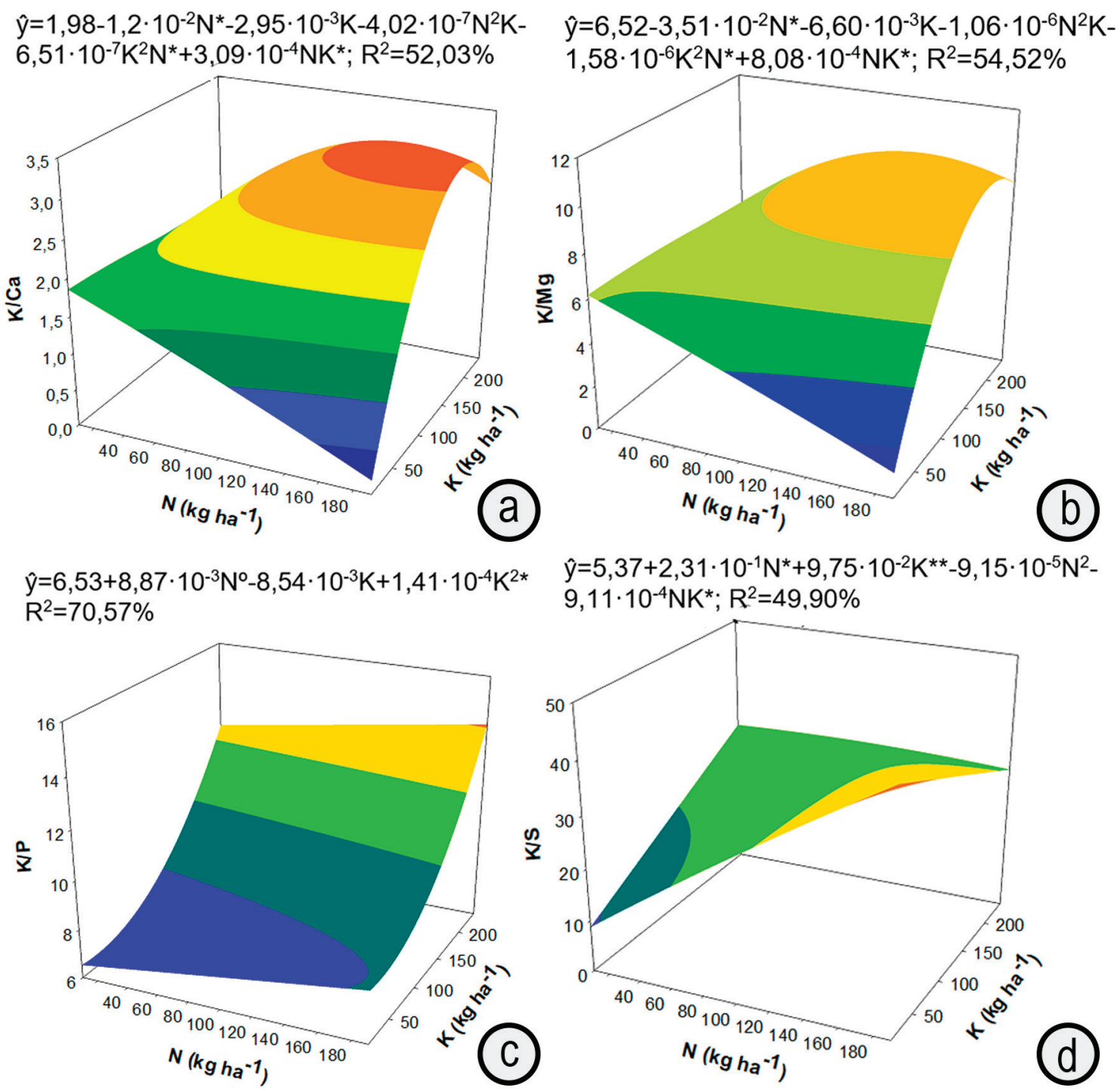

Figura 2. Efecto de dosis de N y K en la relación entre K con: a. calcio; b. magnesio; c. fósforo; d. azufre, en el tejido vegetal de albahaca. 
La respuesta de la relación $\mathrm{K} / \mathrm{P}$ en función de las dosis de $\mathrm{N}$ y $\mathrm{K}$ (Figura 2C) coincide con la de los tratamientos T8, T11 y T12, donde la acumulación foliar de $\mathrm{K}$ sobre $\mathrm{P}$ es favorecida con la adición de $\mathrm{N}$ y $\mathrm{K}$.

En K/S, la mayor relación se registró con el T3 y T12, cuya MS promedio fue de 43,38g, que superó en 155,93\% los valores del T9 y T14, donde la relación de estos nutrientes fue la más baja, con un promedio de 12,82 (Tabla 1 y 3). En esta relación, al igual que para $\mathrm{K} / \mathrm{Ca}, \mathrm{K} / \mathrm{Mg}$ y $\mathrm{K} / \mathrm{P}$, la eficiencia de la ganancia de biomasa precisa de una alta proporción de potasio que, en este caso, debe ser de aproximadamente 46 veces la de azufre.

En la figura 2D, se aprecia que hubo efectos significativos para $\mathrm{N} \mathrm{y}$ $\mathrm{K}$, así como en la interacción $\mathrm{N} * \mathrm{~K}$, respuesta que indica una mayor relación $\mathrm{K} / \mathrm{S}$ con la aplicación de $\mathrm{N}$ y K. Estos resultados difieren de Nurzyńska et al. (2012) en albahaca, donde el S foliar aumentó con la aplicación $\mathrm{N}$ y K.
$\mathrm{Al}$ analizar las correlaciones entre las relaciones de los macroelementos primarios y secundarios con la ganancia de masa seca de hoja (MSH), se encontró que la única relación negativa, para este parámetro, se presentó en la relación N/K (Tabla 4). En estas relaciones, se puede restringir la síntesis de muchas proteínas que son necesarias para formación de estructuras vegetales; además, el potasio, a pesar de encontrarse en altas concentraciones en tejidos vegetales, no hace parte de estructuras vegetativas, pero contribuye con muchos procesos fisiológicos en las plantas. Según Marschner (2012), el K no es un constituyente de las moléculas funcionales o estructuras de la planta, pero está involucrado en numerosos procesos bioquímicos y fisiológicos vitales para el crecimiento de la planta, rendimiento y calidad.

En conclusión, la mayor producción de albahaca en MS se asoció con altas tasas foliares de $\mathrm{N}$ y K en relación con el resto de los nutrientes. La eficiencia en la producción de biomasa de este cultivo, en las condiciones estudiadas, requiere la aplicación de dosis entre 100-190kg ha ${ }^{-1}$ de N y $125-237,5 \mathrm{~kg} \mathrm{ha}^{-1}$ de K.

Tabla 4. Coeficientes de correlación entre las relaciones de algunos nutrientes en el tejido foliar y la masa seca de hojas (MSH) de plantas de albahaca, cultivadas en invernadero.

\begin{tabular}{|c|c|c|c|c|c|c|c|c|}
\hline & $\mathbf{N} / \mathbf{K}$ & $\mathbf{N} / \mathbf{P}$ & $\mathbf{N} / \mathbf{S}$ & $\mathbf{N} / \mathbf{C a}$ & $\mathbf{K} / \mathbf{C a}$ & $\mathbf{K} / \mathbf{M g}$ & $\mathbf{K} / \mathbf{P}$ & $\mathbf{K} / \mathbf{S}$ \\
\hline $\mathbf{M S H}$ & $-0,40^{* *}$ & $0,52 * *$ & $0,50 * *$ & $0,49 * *$ & $0,50 * *$ & $0,41 * *$ & $0,52 * *$ & $0,60 * *$ \\
\hline
\end{tabular}

**: Correlación significativa al 1\% ( $\mathrm{p} \leq 0,01)$.

Conflictos de intereses: Este artículo fue preparado y revisado con la participación de todos los autores, quienes declaramos que no existe conflicto de intereses que ponga en riesgo la validez de los resultados presentados. Financiación: Este estudio fue financiado con recursos del Grupo Regional de Investigación Participativa de los Pequeños Productores de la Costa Atlántica, adscrito a la Universidad de Córdoba.

\section{REFERENCIAS}

1. AGRONET. 2019. Estadísticas agrícolas: área, producción y rendimiento nacional del cultivo de albahaca. Disponible desde internet en: https://www.agronet.gov.co/estadistica/Paginas/home. aspx?cod=1\# (con acceso el 17/05/2019).

2. AZCÓN, J.; TALÓN, M. 2008. Fundamentos de Fisiología Vegetal. Ed. McGraw-Hill (España). p.13-122.

3. BAR-TAL, A.; ALONI, B.; KARNI, L.; ROSENBERG, R. 2001. Nitrogen nutrition of greenhouse pepper. II. Effects of nitrogen concentration and $\mathrm{NO}_{3}: \mathrm{NH}_{4}$ ratio on growth, transpiration, and nutrient uptake. American HortScience. (United States). 36(7):1252-1259.

https://doi.org/10.21273/HORTSCI.36.7.1252
4. BENAVIDES-MENDOZA, A.; HERNÁNDEZ-VALENCIA, R.E.M.; RAMÍREZ, H.; RANGEL, A. 2010. Tratado de botánica económica moderna. Ed. Universidad Autónoma Agraria Antonio Narro (México). 332p.

5. BIESIADA, A.; KUŚ, A. 2010. The effect of nitrogen fertilization and irrigation on yielding and nutritional status of sweet basil (Ocimum basillicum L.). Acta Sci. Pol. Hortorum. (Poland). 9:3-12.

6. BONILLA, C.; GUERRERO, M. 2010. Albahaca (Ocimum basilicum L.): producción y manejo poscosecha. Ed. Corredor Tecnológico Agroindustrial (Colombia). 123p.

7. BOX, G. 1954. The exploration and exploritation of the response surfaces: some general considerations and examples. Biometrics. (United States). 10(1):16-60. https://doi.org/10.2307/3001663

8. CONLEY, M.E.; PAPAROZZI, E.T.; STROUP, W.W. 2002. Leaf anatomical and nutrient concentration responses to nitrogen and sulfur applications in poinsettia. J. plant nutrition. (England \& Walles). 25(8):1773-1791. https://doi.org/10.1081/PLN-120006057 
9. CORTÉS, D.; CLAVIJO, J. 2008. Evaluación agro-fisiológica de la producción de albahaca (Ocimum basilicum L.) bajo invernadero en la sabana de Bogotá. Ed. Universidad Nacional de Colombia (Colombia). 23p.

10. DE SOUZA SILVA, M.L.; TREVIZAM, A.R.; DE CÁSSIAPICCOLO, M.; FURLAN, G. 2014. Tomato production in function of sulfur doses application. Applied Research \& Agrotechnology. (Brasil). 7(1):47-54. https://doi.org/10.5935/PAeT.V7.N1.05

11. DZIDA, K.; ZAWISLAK, G.; KARCZMARZ, K. 2015. Yields and biological value of three herbal species from the Lamiaceae family. J. Elem. (Poland). 20(2):273-283. https://doi.org/10.5601/jelem.2014.19.4.616

12. FERREIRA, S.; BULEGON, L; YASSUE, R.; ECHER, M. 2016. Efeito da adubação nitrogenada e da sazonalidade na produtividade de Ocimum basillicum L. Rev. Bras. Pl. Med. 18(1):67-73. https://doi.org/10.1590/1983-084X/15_035

13. FRABBONI, L.; DA SIMONE, G.; RUSSO, V. 2011. The influence of different nitrogen treatments on the growth and yield of Basil (Ocimum basilicum L.). J. Chem. Chem. Eng. (Germany). 5(9):799-803.

14. INSTITUTO GEOGRÁFICO AGUSTÍN CODAZZI, IGAC. 2006. Métodos analíticos del laboratorio de suelos. Ed. IGAC (Colombia). p.648.

15. MALAVOLTA, E.; MORAES, M.F. 2007. Niquel, de nutriente tóxico a nutriente esencial. Informaciones Agronómicas (Ecuador). 67:10-12.

16. MALIK, A.A.; SURYAPANI, S.; AHMAD, J. 2011. Chemical vs organic cultivation of medicinal and aromatic plants: the choice is clear. Int. J. Med. Arom. Plants. (India). 1(1):5-13.

17. MAPELI, N.C.; VIEIRA, M.D.; HEREDIA, Z.; NÉSTOR, A.; SIQUEIRA, J.M. 2005. Produção de biomassa e de óleo essencial dos capítulos florais da camomila em função de nitrogênio e fósforo. Hortic.Bras. 23(1):32-37. https://doi.org/10.1590/S0102-05362005000100007

18. MARSCHNER, H. 2012. Mineral nutrition of higher plants. third ed. Academic Press. (Australia). 889p.

https://doi.org/10.1016/B978-0-12-473542-2.X5000-7

19. MONCAYO, M.D.R.; ÁLVAREZ, V.D.P.; GONZÁLEZ, G.; SALAS, L.; CHÁVEZ, J.A. 2015. Producción orgánica de albahaca en invernadero en Comarca Lagunera. Terra Latinoamericana. (México). 33(1):69-77.

20. NUKAYA, A.; GOTO, K.; JANG, H.; KANO, A.; OHKAWA, K. 1995. Effect of $\mathrm{NH}_{4} \mathrm{~N}$ level in the nutrient solution on the incidence of blossom-end rot and gold specks on tomato fruit grown in rockwool. In: Ando T.; Fujita K.; Mae T.; Matsumoto, H.; Mori, S.; Sekiya, J. (eds). Plant Nutrition for Sustainable Food Production and Environment. Ed. Springer (Berlin). p.381-388. https://doi.org/10.1007/978-94-009-0047-9_316

21. NURZYŃSKA-WIERDAK, R.; ROŻEK, E.; BOROWSKI, B. 2011. Response of different basil cultivars to nitrogen and potassium fertilization: total and mineral nitrogen content in herb. Acta Sci. Pol. Hortorum. (Poland). 10(4):217-232.

22. NURZYŃSKA-WIERDAK, R.; ROZEK, E.; BOROWSKI, B.; DZIDA, K.; JAROSZ, Z. 2012. Changes in the content of some macronutrients in basil herbage induced by different nitrogen and potassium fertilization rates. J. Elem. (Poland).17(3):465-477. https://doi.org/10.5601/jelem.2012.17.3.09

23. ORMAN, S. 2012. Effects of elemental sulphur and farmyard manure applications to calcareous saline clay loam soil on growth and some nutrient concentrations of tomato plants. J. Food, Agriculture \& Environment. (Finland). 10(2):720725.

https://doi.org/10.5897/AJB11.2701

24. PALENCIA, G.; MERCADO, T.; COMBATT, E. 2006. Estudio Agrometeorológico del departamento de Córdoba. Ed. Gráficas del Caribe (Colombia). 126p.

25. PEDRAZA, R.; HENAO, M.C. 2008. Composición del tejido vegetal y su relación con variables de crecimiento y niveles de nutrientes en el suelo en cultivos comerciales de menta (Mentha spicata L.). Agronomía Colombiana. 26(2):186-196.

26. PUTTANNA, K.; GOWDA, N.N.; RAO, E.P. 2001. Effects of applications of $\mathrm{N}$ fertilizers and nitrification inhibitors on dry matter and essential oil yields of Java citronella (Cymbopogon winterianus Jowitt.). J. Agricultural Science. (United Kingdom). 136(4):427-431. https://doi.org/10.1017/S0021859601008966

27. R CORE TEAM. 2019. R: A language and environment for statistical computing (versión 3.5.3). Disponible desde internet en:

https://www.r-project.org/ (con acceso el 15/02/2019).

28. RAO, E.P.; PUTTTANNA, K.; RAO, R.G.; RAMESH, S. 2007. Nitrogen and potassium nutrition of French basil (Ocimum basilicum Linn.). J. Spices and Aromatic Crops. (India). 16(2):99-105.

29. REICHARDT, K.; TIMM, L.C. 2004. Adsorção de nutrientes pelas plantas. En: Reichardt, K.; Timm, L.C. (eds.). Solo, planta e atmosfera: conceitos, processos e aplicações. Ed. Manole (Brasil). p.341-355. 
30. ROBERTSON, G.P.; VITOUSEK, P.M. 2009. Nitrogen in agriculture: balancing the cost of an essential resource. Annu. Rev. Environ. Resour. (United States). 34:97-125. https://doi.org/10.1146/annurev.environ.032108.105046

31. ROSOLEM, C.A. 2005. Interação do potássio com outros íons. En: Yamada, T; Roberts, T.L. (eds.). Potássio na agricultura brasileira. Ed. UFV (Brasil). p.239-260.

32. RUIZ, H.A. 1997. Relações molares de macronutrientes em tecidos vegetais como base para a formulação de soluções nutritivas. Ceres. (Brasil). 44(255):533-546.

33. SHARAFZADEH, S.; ALIZADEH, O. 2011. Nutrient supply and fertilization of basil. Advances in Environmental Biology. 5:956-960.
34. SHUMAN, L.M. 1994. Mineral nutrition. In: Wilkinson, R.E. (ed.). Plant environment interactions. Ed. Marcel Dekker (New York). p.49-182.

35. XU, Y.W.; ZOU, Y.T.; HUSAINI, A.M.; ZENG, J.W.; GUAN, L.L.; LIU, Q.; WU, W. 2011. Optimization of potassium for proper growth and physiological response of Houttuynia cordata Thunb. Environmental and experimental botany. (Netherlands). 71(2):292-297.

https://doi.org/10.1016/j.envexpbot.2010.12.015

36. YASSUE, R.M.; ACHRE, D.; PASTORIO, M.A.; FERREIRA, S.D.; DETTMER, C.A.; DE MORAES ECHER, M. 2018. Nitrogen fertilization in two varieties of basil. J. Agricultural Science. (United Kingdom). 10:403-408. https://doi.org/10.5539/jas.v10n7p403 